$15^{\text {th }}$ International Conference on

AEROSPACE SCIENCES \& AVIATION TECHNOLOGY,

$\boldsymbol{A S A T}$ - 15 - May 28 - 30, 2013, Email: asat@ mtc.edu.eg,

Military Technical College, Kobry Elkobbah, Cairo, Egypt,

Tel: +(202) 24025292 -24036138, Fax: +(202) 22621908

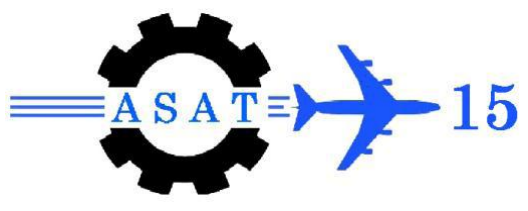

\title{
High Resolution Digital Terrain Elevation Processing
}

\author{
M. Safy ${ }^{*}$, Guangming $\mathrm{Shi}^{\dagger}$, Zhenfang $\mathrm{Li}^{\dagger}$, A.S. Amein ${ }^{\ddagger}$
}

\begin{abstract}
The target of high resolution digital terrain elevation sequence is to obtain 3-D representation of a terrain's surface. In this work we used interferometric technique which is based on the analysis of the phase difference between the signals received at two antenna position. Under suitable conditions, the resulting DEM of this method is locally the most precise one. This work is concerned with the exploitation of radar SLC images of the Las Vega area acquired on 24 May and 25 May 1994, the first is taken as the master image and the other as slave. We have developed an interferometric process beginning with the implementation of a co-registration algorithm for the images. Then we developed the flatearth phase elimination from the interferogram. We also developed a filter in order to minimiz the existing noise. At the end remove the effect of under sampling to get a good result in the final step of the process which is the unwrapping step.
\end{abstract}

Keywords: Image co-registration; Harris corner; RANSAC; interefrogram flattening and interferogram filtering.

\section{Introduction}

There are two basic reconstruction techniques one of which is based on one SAR acquisition and the other is based on two SAR acquisitions. The technique based one SAR acquisition is called radarclinometric technique, Radarcliometry is the science which uses the back diffracted signal from the ground as a measure of the orientation of the part of the ground diffracting the wave with respect to the direction of the emitter. Radarclionometry has proved its efficiency in some historical situations, but was not really used in practical cases despites some experimental and theoretical works [1]. Faced to the radarclinometric method, two other reconstruction techniques exist which are the topic of many current researches. These two methods need two SAR acquisitions of the considered ground. The first one is the Radargrammetry computing elevation from parallax measurements in two overlapping radar images. The radargrammetry technique for DEM generation can provide larger coverage per processing time and software capability than InSAR processing. However the main problem of radargrammetry is that the DEM products have low level quality of DEM due to the Range accuracy of SAR imageries and the terrain slope. The second method is the interferometric technique which is based on the analysis of the phase difference between the signals received at two antenna positions. Under suitable conditions, the resulting DEM of this method is locally the most precise one.

\footnotetext{
*Egyptian Armed Forces, Egypt; msafy_2010@yahoo.com

$\dagger$ Xidian University, Xian, China.

*Egyptian Armed Forces, Egypt; asamein@gmail.com
} 


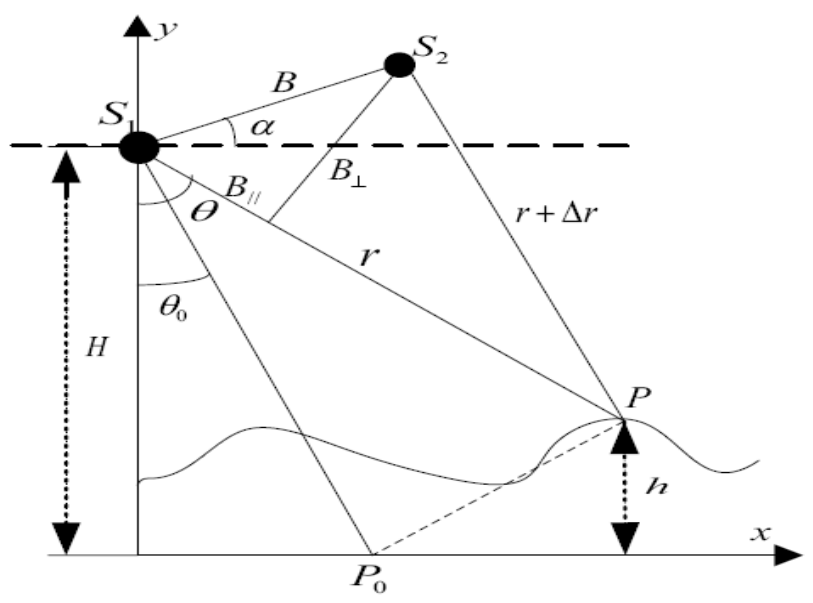

Fig.1. Viewing geometry in airborne SAR interferometry

$\mathrm{S}_{1}$ and $\mathrm{S}_{2}$ are the two antenna positions, $\mathrm{B} / /$ and $\mathrm{B} \perp$ are the horizontal component and vertical component of the baseline, respectively, $\alpha$ is the tilt angle and $\theta_{\mathrm{o}}$ is the incidence angle.

The interferogram is obtained by this interferometric product:

$S_{M} \times S_{S}^{*}=A_{M} \cdot A_{S} \cdot \exp (\Delta \varphi)$

where $S$ is the complex image, and $A$ is the amplitude of the complex image.

$\Delta \phi=-\frac{4 \pi}{\lambda}(r-(r+\Delta r))+\Delta \phi_{\text {erreur }}$

where $r$ is the distance between target and antenna $S_{l}$, perror is the phase error due to signal delay caused by several phenomena (acquisition system, atmosphere etc ....).

We assume that the phase error of the two images is the same especially when the same satellite took the two images (double pass) and the atmospheric conditions are neglected. This phase is divided into a topographic phase proportional to the elevation and a flat-earth phase which is the result of the baseline and the radar acquisition.

In Section 2 we begin with a short literature review which describes our process in detail. In Section 3, we give the experimental results and assess the processing performance. The conclusion is given in Section 4.

\section{Methodology}

In our work we start with The SAR co-registration procedure which consists of 1) coarse coregistration for pixel level accuracy including searching for coarse image offsets and shifting the slave image; 2) fine co-registration for subpixel accuracy which includes collecting salient points from each Image, selecting correspondences between points and removing Outliers using Random Sample Consensus (RANSAC) Algorithm. Then co-registration performance is evaluated using coherence factor.

Then we use Fast Fourier transform to detect the peak of frequency shift to eliminate the flatearth phase from the interferogam, and to make the result more accurate we use oversampling 
technique to detect the decimal value of the frequency shift peak position. We use vector filtering method to minimize the interferogram existing noise. Immerkaer method [2] is used as the evaluation method and to calculate the noise variance before and after filtering. At the end remove the effect of under-sampling, which is elementary to unwrap the final phase. Figure 2 illustrates the main steps of the process.

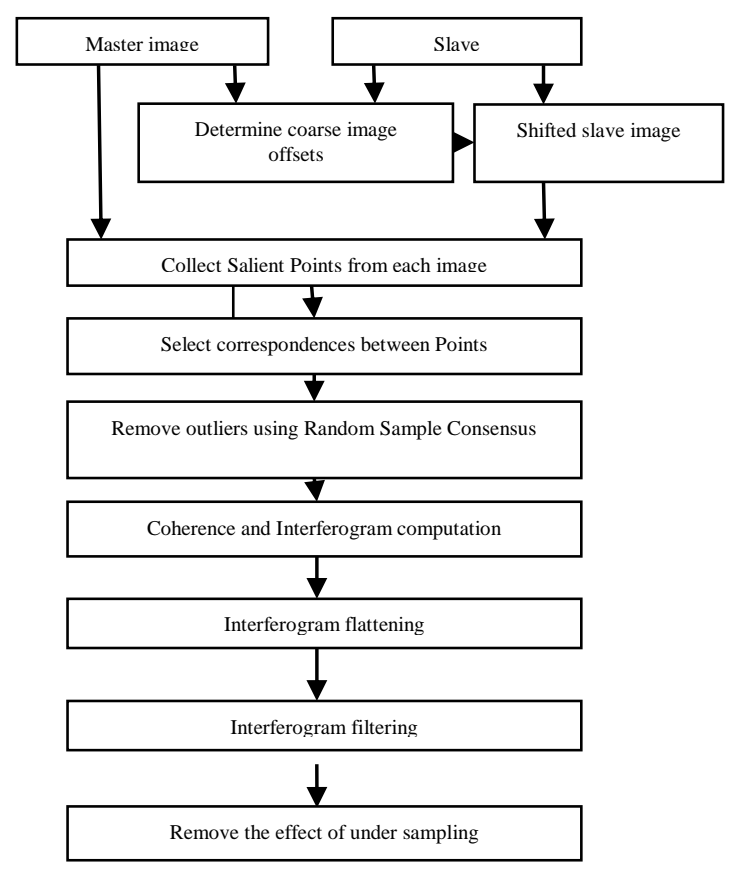

Fig.2. Main steps of the process

\subsection{Image Coregistration}

Interference requires pixel to pixel match between common features in image pairs. Thus coregistration, the alignments of SAR images from two antennas, is an essential step for the accurate determination of phase difference and for noise reduction.

\subsubsection{Coarse co-registration}

Coarse co-registration is the step where two SAR images are co-registered at up to one or two pixels accuracy. One of these two SAR images must be assigned as the master (reference) image and another one is the slave (match) image. Usually the image located closer to the target of interest is selected as the master image to provide the best geometry. Crosscorrelation (Li and Goldstein, 1990; Liao et al., 2004) is the most commonly used approach for coarse coregistration. The algorithm is simple to implement with the speed and accuracy and it is data insensitive and can be applied in automatic InSAR processing easily. Crosscorrelation can be calculated in frequency domain for the faster processing. After all patch pairs with good cross-correlation are finalized, the average peak coordinates are computed and regarded as range and azimuth offsets between two SAR images. The slave SAR image will be shifted by the range and azimuth offsets.

\subsubsection{Fine co-registration}

After coarse co-registration one has the original master SAR image and the shifted slave SAR image. The misregistration between them now is just one or two pixels. In order to obtain an accurate InSAR phase difference, subpixel co-registration is required for SAR images. That is the fine co-registration. 
The misalignment between slave and master images appears more in edges than in the homogeneous regions. Therefore it is better to find interesting points instead of all the edge points to estimate the transformation. There are many interest point detectors available and evaluated to detect features [3]. But it has been found that Harris corner detector gives better performance. Harris corner detector has strong invariance to rotation, scale, illumination variation and image noise. Harris corner detector is based on the local auto-correlation function of a signal, where the local auto-correlation function measures the local changes of the signal with patches shifted by a small amount in different directions [4].

The basic idea of Harris corner detector is to give a mathematical approach for determining whether a point shows significant change in all the directions to designate as a corner point. The point can be easily recognized by looking at intensity values within a small window and shifting the window in any direction which should yield a large change in appearance.

We use the normalized cross-correlation between the two images regions as well as the matching cost between points. Once the control point match pairs have been found, the next essential step is to determine a spatial transformation model that maps the control points from the slave image to the corresponding points in the master image. The most common type of transformation models used for satellite image registration is simple translation, rigid, projective, affine, and polynomial transformation. However the major issue in this step is to estimate the parameters of the selected transformation model. The conventional approach is to estimate the model parameters using least square estimate. However, the main problem of least square estimate is that its performance degrades in the presence of outliers. This is why Random Sample Consensus [5] (RANSAC) is applied to such problems due to its simple implementation and robustness.

\subsection{Interefrogram Generation}

The SAR interferogram is generated by cross-multiplying the first SAR image with the complex conjugate of the second pixel by pixel [Bamler98A, Massonnet98, Franceschetti99]. Thus the interferogram amplitude is the amplitude of the first image multiplied by that of the second one, whereas its phase (the interferometric phase) is the phase difference between the images.

\subsubsection{Interferogram flattening}

During the data processing, for the imaging geometry, the phase difference will exist between the two scattering elements locating on the same height, and phases in the interferogram often parallel to the azimuth or slant aspect, which is defined as flat-earth phase effect. It is very important to remove the flat-earth phase in order to decreases the phase density and reduce the complexity of unwrapping $[6,7,8]$.The accuracy of flat-earth removal will influence the further process as it will make the phase to DEM transformation more difficult for the nonlinear function and Ground Control Points (GCP) requirement. The main goal of flattening is to wipe out the flat earth phase which has nothing with topographic elevation or surface deformation [8].

When there is no deformation, original phase difference can be expressed as:

$\phi=\phi_{\text {topo }}+\phi_{\text {flat }}+\phi_{\text {noise }}$

If surface deformation takes place during the imaging times span, original interferometric phase will be characterized as $[9,10]$ : 
$\phi=\phi_{\text {deformation }}+\phi_{\text {topo }}+\phi_{\text {flat }}+\phi_{\text {atm }}+\phi_{\text {noise }}$

The purpose of flattening is to remove $\boldsymbol{\varphi}$ flat in the interferogram. We use Fast Fourier transform to detect the frequency-shift peak which usually determines the maximum fringe frequency in the range direction where the flat-earth phase is dominant. It is removed by corresponding linear phase compensation in the time domain (by complex multiplication) or the frequency domain (by circular shift).

For conventional frequency-shift method only integer samples of frequency deviation can be easily detected and compensated to the zero frequency, so we use oversampling technique to find the decimal value of the frequency-shift peak. This will lead to accurate definition for the frequency-shift peak.

\subsubsection{Interferogram filtering}

The filtering of interferogram has great influence on phase unwrapping, There are three interferogram filtering methods:

a) Non-adaptive filtering methods: These methods include mean filtering, median filtering, and so on. All of them are effective for optical images but not for INSAR interferograms. These methods adopt some fixed windows for filtering and can induce the distortion of phases due to the periodicity character of interferogram that is not considered.

b) Adaptive filtering methods (Jong-Sen Lee,1998): These methods is aiming at the orientation of the fringe, and uses directionally dependent windows to filter noises along the fringes, but it requires phase unwrapping in the operating window before filtering and phase rewrapping after filtering. In addition it is a lot of work to select the directional window plate. Hence it is not so effective for filtering of interferogram because of difficulty of phase unwrapping and its slow processing.

Multilooking filtering method is a common filtering method for interferogram. This filtering method is very effective for fringe filtering and is used more and more. Supposing $\phi_{1}$ and $\phi_{2}$ are the phases with noises, whose energies are respectively $A_{1}$ and $A_{2}$, but $A_{2}$ is much higher than $A_{1}$, so the result phase $\phi$ will be close to $\phi_{1}$. So we can find that if the energy of the noise is much higher than that of others, the filtering result can be affected to lower precision.

Due to the disadvantages of the previous methods we use in our work Vector Filtering. Vector Filtering is a new filtering method for interferogram fringes. Given an interferogram image, the vector filter spans the following steps:

1. Calculate the sine and cosine for each pixel.

2. Filter the sine and cosine images

3. Calculate the interferogram again using the arctangent function

This procedure explores the continuity of the sine and cosine functions to avoid discontinuity in the interferogram image.

Filtering in step 2 is done using any filter, but this is also a crucial step, because the chosen filter must not degrade the quality of the phase. We have to keep the statistical parameters of the pixel as much as possible. 


\subsection{Removing the Effect of Under-Sampling}

Under-sampling is essentially sampling too slowly, or sampling at a rate below the Nyquist frequency for a particular signal of interest. Under-sampling leads to aliasing and the original signal cannot be properly reconstructed. Phase unwrapping of phase images that are under sampled can be difficult, or in some cases even impossible. So we must detect if the sampling rate in range and azimuth direction is sufficient or not. Calculate the maximum phase change in range and azimuth direction respectively, divide it by $\pi$ because successive samples in the range and azimuth directions must be change by a value of less than $\pi$ and multiply the result by the value of range and azimuth respectively.

\section{Data, Tools and Experiments}

\subsection{Data and Tools}

SAR pairs of ERS-1/2 tandem mode single look complex images were used. The pair consists of one ERS-1 image acquired on May 23, 1996 and other ERS-2 image acquired on May 24, 1996. This pair covers Las Vegas in the USA. Matlab is the main tool that was employed for SAR image co- registration and coherence computation. Gamma Design software is used to compare the result with the proposed co -registration algorithm.

\subsection{Results}

\subsubsection{Proposed image co-registration algorithm}

a) Coarse co-registration using the cross correlation function. The offset in range is 199 and in azimuth is 6 and the coherence before co-registration is 0.3933 while the coherence after coarse co-registration is 0.5251 .

b) Fine co-registration using feature based method (Harris corner detector and RANSAC algorithm).The offset in range is -0.3119 and in azimuth is 0.00286 and the coherence after fine co-registration is 0.5398 .

Table 1 illustrates the difference in coherence factor between the proposed algorithm, feature based method and Gamma design software.

Table 1 Coherence factor

\begin{tabular}{l|c}
\hline \hline \multicolumn{1}{c|}{ Process name } & Coherence factor \\
\hline Feature based method & 0.5147 \\
\hline Gamma design software & 0.5435 \\
\hline Proposed algorithm & 0.5398 \\
\hline \hline
\end{tabular}

In the following figures we present the results of the co-registration experiments. 


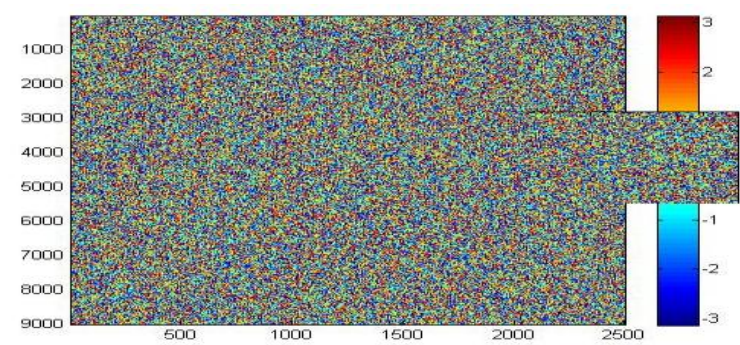

Fig.3 Interferogram before coregisteration.

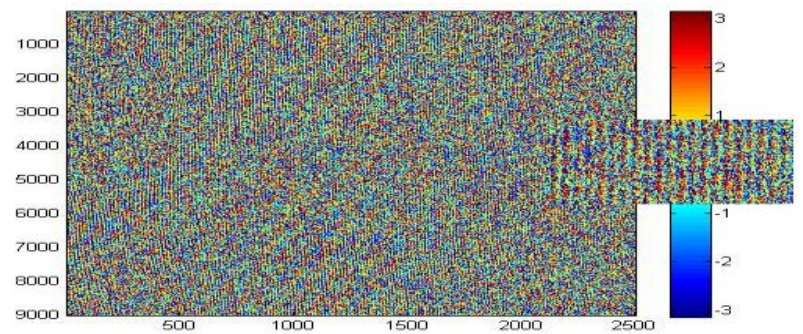

Fig.4 Interferogram after fine coregisteration.

\subsubsection{Intereferogram generation}

By using Fast Fourier transform the frequency shift peak is 1041 and after using oversampling technique we found that the shift is 1040.6. In this work we used different types of filters to choose the best filter suitable for this type of lands Table 2 illustrates the noise variance estimation after using the different types of filters.

Table 2 Noise variance estimation after using the different types of filters.

\begin{tabular}{l|l}
\hline \hline Filtering Method & Noise variance \\
\hline Vector method using median filter & 0.6556 \\
\hline Vector method using adaptive median filter & 1.2914 \\
\hline Vector method using mean filter $(3 \times 3)$ & 0.6223 \\
\hline Vector method using mean filter $(7 \times 7)$ & 0.2055 \\
\hline Vector method using mean filter $(13 \times 13)$ & 0.0910 \\
\hline Vector method using mean filter $(17 \times 17)$ & 0.0681 \\
\hline Vector method using mean filter $(25 \times 25)$ & 0.0473 \\
\hline \hline
\end{tabular}

In the following figures we present the results of the inteferogram generation experiments.

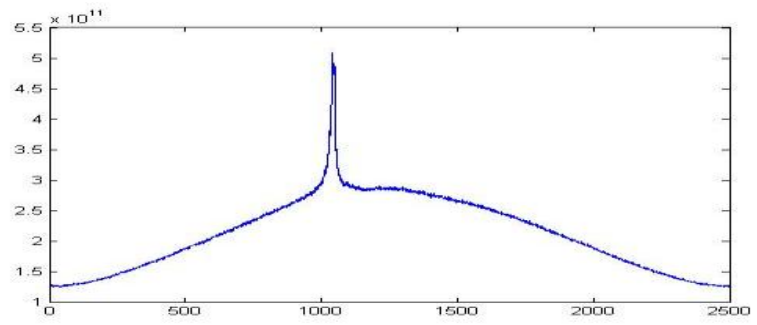

Fig. 5 Frequency shift peak before Interferogram flattening. 


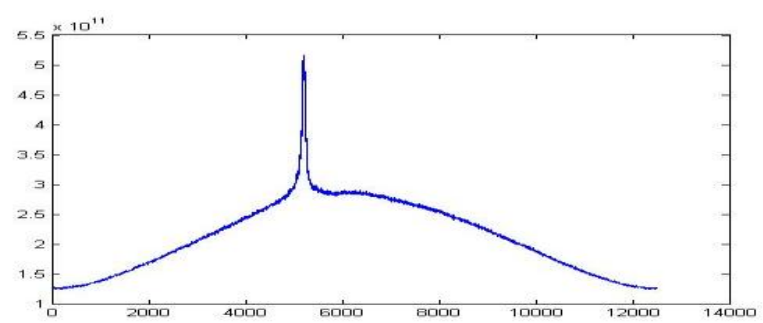

Fig. 6 Frequency shift peak before Interferogram flattening in case of oversampling.

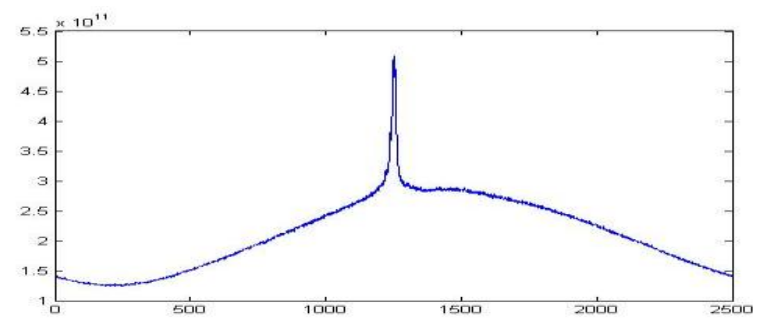

Fig.7 Frequency shift peak after interferogram flattening.

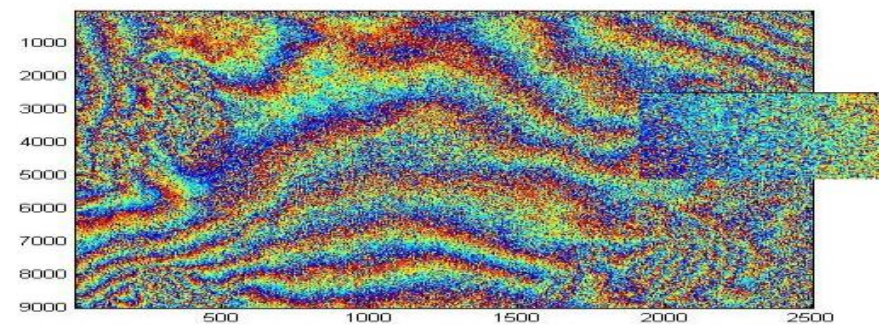

Fig. 8 Interferogram flattening.

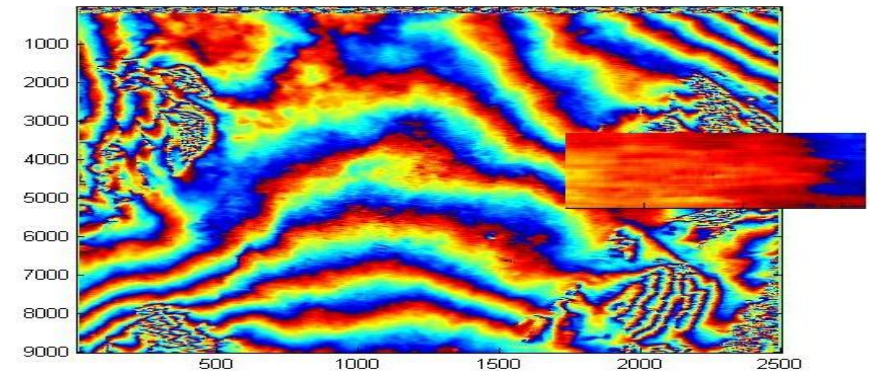

Fig. 9 Interferogram filtering.

\subsubsection{Removing the effect of under-sampling}

The location of the maximum phase change in the range direction is $x=1511$ and $y=331$ and for azimuth is $\mathrm{x}=686 \mathrm{and} \mathrm{y}=8086$. The value of this maximum phase change at these locations for range and azimuth is 6.2828 radians and the sampling rate is 2 in the two directions. The number of actual data points sampled in the range direction has to be least 5000 samples and in azimuth direction it has to be at least 18000 samples. To remove the under-sampling, many different resampling schemes are possible. In the following figures we present the max phase change in range and azimuth directions and the oversampled interferogram. 


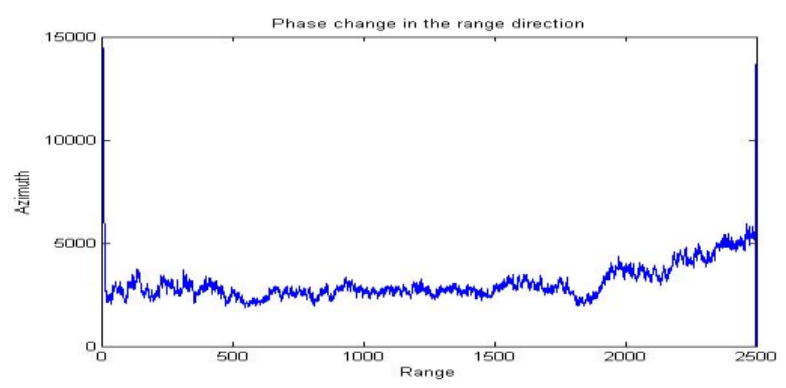

Fig. 10 Maximum phase change in range direction

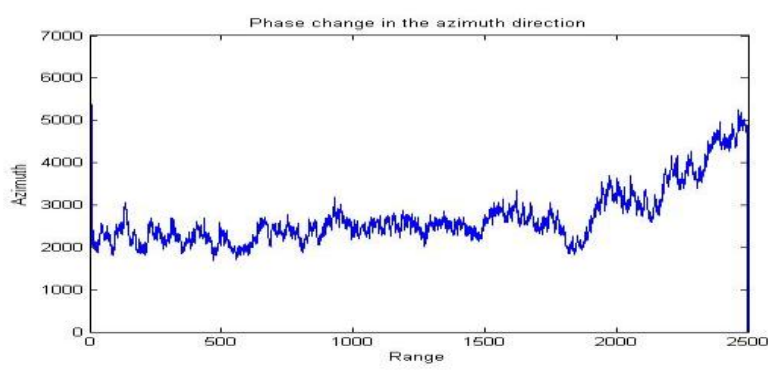

Fig. 11 Maximum phase change in azimuth direction

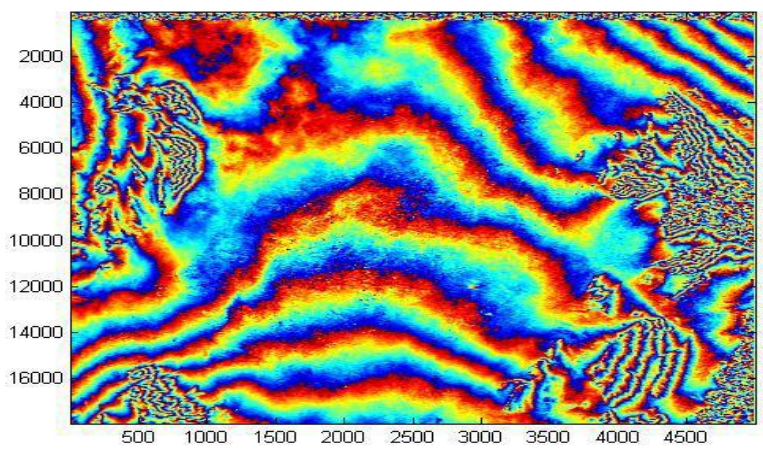

Fig. 12 Oversampled interferogram after filtering

\section{Conclusion}

Our work describes how to develop a part of the interferometric process which is useful for generating a digital elevation model (DEM).We started by co-registeration between the two images using semi automatic algorithm which combines the typical method and feature based method. Then we generated the interferogram by using Fast Fourier transform to eliminate the flat-earth phase from the interferogam .We also used vector filtering method by different filter types and also by different window size to minimizing the noise .At the end remove the effect of under sampling which will be ready to the phase unwrapping step. 


\section{References}

[1] R. Frank and R. Chellappa. Estimation of surface topography from SAR imagery using shape from shading techniques Artificial Intelligence, (43):271-310, 1990.

[2] Immerkaer, John. "Fast noise variance estimation." Computer Vision and Image Understanding 64.2 (1996): 300-302.

[3] Cordelia Schmid, Roger Mohr And Christian Bauckhage," Evaluation of Interest Point Detectors", International Journal of Computer Vision 37(2), 151-172, 2000.

[4] C. Harris and M.J. Stephens,"A combined corner and edge detector", In Alvey Vision Conference, pages 147-152, 1988.

[5] M.A. Fischler and R.C. Bolles. Random sample consensus: A paradigm for model fitting with applications to image analysis and automated cartography. Communications of the ACM, 24(6):381 395, 1981.

[6] Yang Lei, Zhao Yongjun1, Wang Zhigang. (2004). Frequency Shifted - based Approach for InSAR Flat Earth Effect Removal. JOURNAL OF ELECTRONIC MEASUREMENT AND INSTRUMENT, 18 (4): 15-20.

[7] ZHANG Yanjie. (2005). A Comparison of the Different Models Used For Interferograms Flattening. International Geoscience and Remote Sensing Symposium (IGARSS) 5: 5194-5196.

[8] ZENG qiming, LI xiaofan, GAO liang, (2006) An Improvement to Flattening in Interferometric SAR Processing. Proc. of SPIE. 6200: 62000D-1-62000D-6.

[9] Gatelli, A.M. Guarnieri, F. Parizzi, et al, (1994). "The wavenumber shift in SAR interferometry," IEEE Trans. Geosci. Remote Sensing, 32 (4):. 855-865.

[10] R. Siegmund, M. Bao S. Lehner, et al, (2004). "First demonstration of surface currents imaged by hybrid along- andcross-track interferometric SAR," IEEE Trans. Geosci. Remote Sensing, 42 (5): 511-519. 